\title{
Belgeo
}

Revue belge de géographie

4 | 2017

La Belgique : quelques nouveaux regards sur un pays en forte mutation - Belgium: some new looks at a higly evolving country

\section{Bruxelles et ses déplacements domicile-travail et domicile-école}

Thomas Ermans, Céline Brandeleer, Caroline d'Andrimont, Michel Hubert et Pierre Marissal

\section{(2) OpenEdition}

\section{Journals}

Édition électronique

URL : http://journals.openedition.org/belgeo/20506

DOI : 10.4000/belgeo.20506

ISSN : 2294-9135

Éditeur :

National Committee of Geography of Belgium, Société Royale Belge de Géographie

Référence électronique

Thomas Ermans, Céline Brandeleer, Caroline d'Andrimont, Michel Hubert et Pierre Marissal, «Bruxelles et ses déplacements domicile-travail et domicile-école », Belgeo [En ligne], 4 | 2017, mis en ligne le 15 décembre 2017, consulté le 05 mai 2019. URL : http://journals.openedition.org/belgeo/20506 ; DOI : 10.4000/belgeo.20506

Ce document a été généré automatiquement le 5 mai 2019.

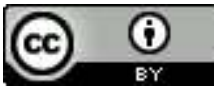

Belgeo est mis à disposition selon les termes de la licence Creative Commons Attribution 4.0 International. 


\title{
Bruxelles et ses déplacements domicile-travail et domicile-école
}

\author{
Thomas Ermans, Céline Brandeleer, Caroline d'Andrimont, Michel Hubert \\ et Pierre Marissal
}

\section{Introduction}

1 Première entité urbaine de Belgique en termes de population et d'emploi, Bruxelles constitue un nœud de transport très important au niveau belge voire international. C'est la raison pour laquelle cet article propose de se focaliser sur cette centralité et de la traiter à travers la mobilité quotidienne en lien avec Bruxelles, sous l'angle de la spatialité et de la temporalité de deux motifs spécifiques de déplacements - les déplacements domicile-travail (DDT) et domicile-école (DDE) -, traditionnellement considérés comme déterminants pour la forme et le dimensionnement des réseaux de transport. Au-delà de l'objet d'étude proprement dit, cet article soulève également un enjeu méthodologique important, à savoir la rupture dans la continuité des données sur les DDT/DDE suite à la disparition, en Belgique, des recensements classiques (par enquête à visée exhaustive) de la population et du logement. Avec le passage au Census, «recensement » basé sur des données administratives, l'information sur la mobilité des travailleurs et des écoliers s'est sensiblement appauvrie. Comme nous l'expliquerons, nous tentons ici de recomposer une image aussi riche que possible de la situation à partir de multiples sources de nature diverse.

2 Les déplacements domicile-travail et domicile-école ne représentent bien sûr qu'une partie des déplacements en lien avec la Région de Bruxelles-Capitale (RBC). Pour la population active et les personnes scolarisées, ils constituent néanmoins l'épine dorsale des routines de déplacements quotidiens, notamment parce que leur réalisation est fortement contrainte spatialement (le plus souvent, entre le ou les lieux de domicile et le ou les lieux de travail ou de scolarité) et dans le temps (horaires relativement fixes).

3 Les routines d'activités et de déplacements que chaque individu tend à mettre en place sont conditionnées par des contraintes individuelles, familiales (composition du ménage, 
présence d'enfants, etc.) et sociétales (horaires de travail, jours fériés, horaires d'ouverture des commerces, etc.). Par routinisation des pratiques de déplacement, on entend dès lors la recherche progressive d'un optimum dans l'organisation quotidienne, permettant également de mobiliser un répertoire d'actions diversifié face à une série de décisions à prendre pour coordonner les emplois du temps. Il ne s'agit pas d'habitudes figées, mais d'une gestion souvent complexe, tant dans le temps que dans l'espace, quand il s'agit de combiner les déplacements professionnels, la gestion des enfants, la flexibilisation des agendas professionnels, les activités récréatives, etc. (Montulet et al., 2007 ; Pradel et al., 2015).

4 La temporalité spécifique, et souvent contrainte, des DDE/DDT donne lieu - on le sait - à une concentration importante des flux sur certaines tranches horaires. Elle rythme dès lors les temps sociaux, y compris pour toutes celles et ceux qui ne sont pas directement concernés par ces motifs. Si la flexibilisation du travail (croissance des horaires décalés, atypiques ou irréguliers) peut contribuer à une plus grande dispersion des flux, elle nécessite par contre une meilleure offre de transport en dehors des heures de pointe et, pour les individus et les ménages, rend parfois plus complexe encore l'organisation de la vie quotidienne et met sous pression leur organisation spatio-temporelle (Montulet \& Hubert, 2008 ; Juan, 2015).

5 Pour traiter des DDT et des DDE en lien avec Bruxelles, nous présenterons d'abord les données disponibles et l'échelle d'analyse privilégiée. Nous rendrons compte ensuite du poids de ces déplacements dans la mobilité en général, relativement aux autres motifs et selon les différents types de jours, de même que nous examinerons l'intensité des déplacements selon différentes tranches horaires. Nous dresserons alors un état des lieux en termes de populations concernées, de volumes de déplacements et de choix modal pour ces motifs, en mettant ces éléments en perspective avec les distances parcourues et leur évolution. Enfin, en conclusion, nous évoquerons les tensions et contraintes que peuvent représenter ces déplacements sur les routines quotidiennes des individus et des ménages.

\section{Sources et échelle d'analyse}

\section{Sources disponibles}

6 L'étude des déplacements domicile-travail a débuté fin du $19^{\mathrm{e}}$ siècle dans plusieurs pays européens (Belgique, Pays-Bas, France, Allemagne...) au travers de recensements permettant de quantifier, à une échelle spatiale fine et au niveau individuel, le lien entre lieux de résidence et de travail (Dickinson, 1957). Cet effort de quantification d'un phénomène qui s'était amplifié tout au long du siècle peut être rattaché à l'émergence de la problématique du déséquilibre dans les études urbaines. En effet, l'objet d'étude derrière ce dispositif de collecte de données était moins le phénomène des déplacements domicile-travail en soi (et par là également le phénomène de migration alternante) que le fait qu'il puisse être symptomatique d'un déséquilibre dans la répartition territoriale des lieux de résidence et de travail. Cette disparité territoriale, ayant notamment pour effet la saturation des réseaux de transport, prend une véritable importance dans le débat public à partir des années 1950. L'objet de la quantification des déplacements domicile-travail devient alors également une mesure "dimensionnante» permettant de calibrer les 
infrastructures de transport à l'importance des migrations alternantes à l'heure de pointe (Commenges, 2013).

7 L'abandon des recensements après 2001 et leur remplacement par un recensement «administratif », le Census (2011), interrompt la série temporelle et entrâne une recomposition de l'offre de données. L'association des sources Census et de l'Enquête sur les Forces de Travail (EFT), que nous avons réalisée ici, permet de retrouver à la fois un certain degré de désagrégation des recensements classiques pour le traitement des flux «bruts» et un éventail de concepts adaptés à la mesure de la mobilité, disponibles seulement à un niveau plus macro. En effet, réalisée en continu sur un échantillon annuel de 80.000 personnes de plus de 15 ans domiciliées sur le territoire belge, l'EFT présente l'intérêt de produire des variables conceptuellement très proches de celles des recensements classiques, ce qui permet de perpétuer dans une certaine mesure la série temporelle.

Les déplacements domicile-école sont, de leur côté, nettement moins bien documentés dans le temps. Ils ne seront repris de façon systématique, au travers du recensement, qu'à partir de l'édition 1991. Aujourd'hui, les principales données disponibles sont celles qui concernent la situation des élèves scolarisés dans l'enseignement (maternel, primaire et secondaire) subventionné ou organisé par les Communautés (flamande et française). Elles ne comprennent donc pas de données sur l'enseignement privé (non subventionné par les pouvoirs publics) ou les écoles européennes, par exemple. A ces données s'ajoutent toutefois celles des Plans de déplacements scolaires et des prédiagnostics scolaires dont la collecte, bien qu'imparfaite, permet l'analyse par mode des déplacements domicile-école au niveau de l'implantation scolaire ou de l'établissement principal pour les élèves (enseignement maternel et obligatoire) scolarisés en Région de Bruxelles-Capitale.

Enfin, l'enquête nationale BELDAM (2010) sur les pratiques quotidiennes de déplacement des ménages constitue une mine d'informations pertinentes relativement à la mobilité pour l'ensemble du territoire belge. Si, individuellement, chaque déplacement peut y être replacé assez finement dans son contexte spatial, l'échelle d'analyse est bornée au niveau macro en raison de la taille de l'échantillon.

\section{Échelle d'analyse : les déplacements « en lien avec Bruxelles »}

L'histoire du développement urbain bruxellois est caractérisée par un mouvement centrifuge en termes d'habitat. La périurbanisation et l'exode des ménages bruxellois se confirme encore aujourd'hui, bien que celui-ci soit compensé par l'immigration externe et un taux de natalité important au sein de la Région de Bruxelles-Capitale (RBC). Le solde migratoire interne négatif est d'ailleurs assez stable, autour de 13.000 personnes par an, depuis 2004 (Hermia, 2016). Cette tendance à l'étalement urbain implique l'existence d'une certaine densité de population au-delà des frontières administratives de la capitale. Plusieurs communes de la périphérie proche atteignent d'ailleurs une densité de population similaire à celle de certaines communes bruxelloises (figure 1). 
Figure 1. Densité de population au sein de la Zone RER de Bruxelles.

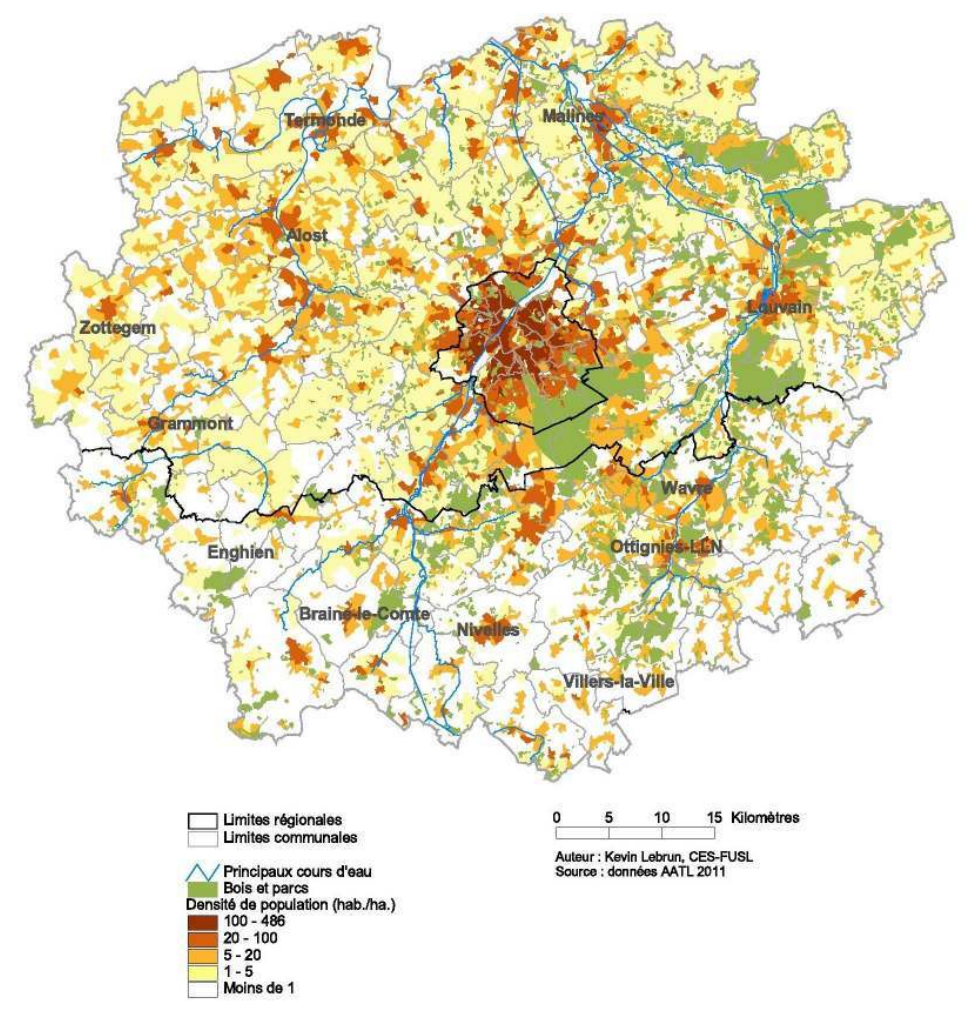

Source: données AATL (2001). Cartographie: Kevin Lebrun (CES - USL-B)

11 En termes de volumes de déplacements, l'enquête BELDAM (2010) nous apprend qu'un jour moyen, les déplacements internes à la RBC représentent les deux tiers des déplacements en lien avec Bruxelles. Bien que cette proportion varie sensiblement selon le type de jour, cela signifie que, toujours pour un jour moyen, un déplacement sur trois en lien avec Bruxelles est un déplacement entrant ou sortant (Lebrun et al., 2013, pp. 9-12). On le voit, une analyse spatiale de la mobilité ne peut se restreindre aux limites administratives de la Région. C'est pourquoi, il est plus pertinent de prendre comme base d'analyse l'ensemble des déplacements «en lien avec Bruxelles ", en distinguant quand c'est possible et pertinent les déplacements entrants, sortants et internes à la RBC.

En matière de vocabulaire, le terme navette renvoie aux personnes qui effectuent un déplacement domicile-travail ou domicile-école qui franchit un certain seuil, typiquement les limites communales (voir notamment INS, 1981 ; Mérenne-Schoumacker et al., 1999 ; Verhetsel et al. 2009 ; Census 2011). Cette terminologie implique également une certaine régularité dans le déplacement, définie par la nécessité de devoir se déplacer au moins quatre jours par semaine (INS, 1981 ; Mérenne-Schoumacker et al., 1999), qui souligne de la sorte la composante «quotidienne» qui s'attache au concept. Dans cet article, nous réserverons le terme de navette aux personnes se déplaçant vers leur lieu de travail ou d'étude s'ils entrent (navette entrante) ou sortent (navette sortante) de la RBC, quel que soit le mode de transport utilisé : c'est donc le franchissement de la frontière administrative régionale qui importe ici. 


\section{Poids des déplacements domicile-travail et domicile- école dans la mobilité quotidienne en lien avec Bruxelles}

\section{Déplacements domicile-travail et domicile-école par rapport aux autres motifs}

Relativement aux autres motifs de déplacement, le motif « aller au travail » compte pour $17,6 \%$ des déplacements en lien avec la Région de Bruxelles-Capitale. On pourrait s'attendre à un chiffre plus élevé mais il s'agit ici d'un jour moyen, qui englobe le samedi, le dimanche et les jours fériés. Cela relativise le poids de ces motifs dans l'ensemble des déplacements. Mais, comme l'indique le Tableau 1, lors des jours ouvrables scolaires, $30,9 \%$ du total des déplacements en lien avec la RBC sont directement motivés par le travail ou l'école. Si on les additionne aux retours à la maison, ce sont environ deux tiers des déplacements qui sont liés au travail ou aux études ces jours-là, ce qui souligne leur caractère particulièrement structurant.

Tableau 1. Distribution des motifs de déplacement en lien avec la RBC selon le type de jour.

\begin{tabular}{|l|l|l|l|l|}
\hline & $\begin{array}{l}\text { Jour ouvrable } \\
\text { scolaire }\end{array}$ & $\begin{array}{l}\text { Jour ouvrable } \\
\text { non scolaire }\end{array}$ & Samedi & $\begin{array}{l}\text { Dimanche et } \\
\text { jour férié }\end{array}$ \\
\hline $\begin{array}{l}\text { Motifs en lien direct avec le } \\
\text { travail ou l'école }\end{array}$ & $30,9 \%$ & $26,8 \%$ & $7,1 \%$ & $9.5 \%$ \\
\hline Autres motifs & $30,6 \%$ & $36,3 \%$ & $51,4 \%$ & $49,7 \%$ \\
\hline Aller à la maison & $38,5 \%$ & $36,9 \%$ & $41,5 \%$ & $40,7 \%$ \\
\hline Total & $\mathbf{1 0 0 \%}$ & $\mathbf{1 0 0} \%$ & $\mathbf{1 0 0} \%$ & $\mathbf{1 0 0 \%}$ \\
\hline \# déplacements & 2.891 & 641 & 531 & 498 \\
\hline
\end{tabular}

Source : BELDAM 2010 (Lebrun et al., 2013 : 12)

\section{Répartition horaire des déplacements}

Les déplacements ne se répartissent pas de manière uniforme sur une journée mais tendent à se concentrer sur certaines plages horaires, appelées communément heures de pointe, qui ont une importance non négligeable sur la saturation des réseaux de transport. En ce qui concerne la contribution des motifs " travail/école » aux heures de pointe, l'heure de départ dépend fortement de l'heure du début du travail ou des cours, mais également de la distance et des activités éventuelles effectuées en cours de route. Il en va de même pour l'heure d'arrivée (Verhetsel et al., 2009, p. 35).

L'enquête socio-économique de 2001 avait confronté les heures de départ et d'arrivée des travailleurs. On avait pu y constater que plus les personnes habitent loin des zones 
urbaines, plus elles tendent à partir tôt pour rejoindre leur lieu de travail. Les différences étaient significatives entre les communes de la périphérie bruxelloise (départs entre $6 \mathrm{~h}$ et 7h30) et les travailleurs bruxellois (entre 8h et 8h30) (Verhetsel et al., 2009, pp. 36-39). Plus actuelle, l'enquête BELDAM (2010) permet également de donner quelques indications sur les horaires de déplacements des travailleurs, mais également des élèves. Les résultats de l'enquête confirment que l'heure de pointe du matin est plus précoce pour les déplacements en lien avec Bruxelles ( $6 \mathrm{~h}$ et $7 \mathrm{~h}$ ), mais indique également des variations importantes d'intensité relative de déplacement selon le type de jour (figure 2). Ainsi, pour les déplacements en lien avec Bruxelles, les pointes du matin et du soir sont davantage marquées pour un jour ouvrable scolaire que pour un autre type de jour (Lebrun et al., 2013, p. 17). C'est particulièrement vrai le matin où l'intensité relative des déplacements en lien avec la Région fait plus que doubler, pour les départs compris entre 7 et $8 \mathrm{~h}$, un jour ouvrable scolaire par rapport à un jour de congé scolaire. Cela a pour conséquence d'augmenter à la fois la durée des trajets (congestion) et leur pénibilité (saturation des transports en commun).

Figure 2. Intensité relative des déplacements en lien avec la RBC, par heure de départ, selon le type de jour, tous motifs confondus.

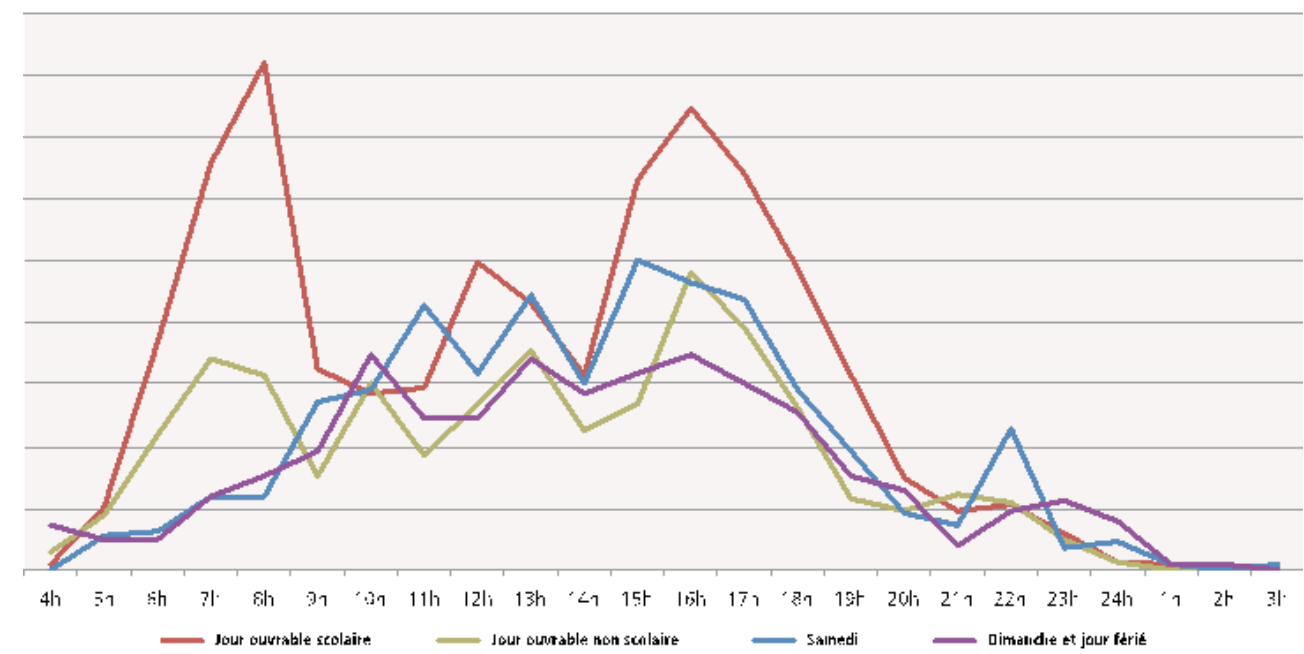

Source : BELDAM 2010

Ce constat est d'autant plus important que la masse des déplacements scolaires est appelée à croître encore dans le contexte de forte croissance démographique que connaît la Région bruxelloise depuis le début des années 2000.

\section{Caractéristiques et tendances}

\section{Volumes et fréquences de déplacements}

17 A l'issue d'une analyse approfondie des données (voir Ermans et al., à paraître), nous estimons que plus de 1040000 personnes sont concernées en moyenne par les déplacements domicile-travail ou domicile-école en lien avec la RBC. Environ 561000 personnes travaillent ou sont scolarisées dans la Région et y résident, 399000 font de même sans y résider (navette entrante) et 79000 Bruxellois sortent des frontières régionales dans le cadre de leurs activités scolaires ou professionnelles (navette sortante). 
Ces chiffres ne tiennent pas comptent des étudiants de l'enseignement supérieur (près de 100000 à Bruxelles (Vaesen \& Wayens, 2014), sans compter les Bruxellois qui étudient à l'extérieur de la ville, notamment à Leuven et à Louvain-la-Neuve) dont les déplacements sont mal connus. Ils sous-estiment donc la réalité.

L'importance de la population concernée par ces motifs se traduit évidemment en termes de volumes de déplacements. Pour un jour ouvrable moyen, le travail et l'enseignement (à l'exclusion du supérieur) généreraient pas moins de 1640000 déplacements en lien avec la RBC. La majorité de ces déplacements se font en interne (903 000 déplacements), mais la navette entrante en constitue une part substantielle (614 000 déplacements), alors que la navette sortante, plus modeste, est loin d'être négligeable (123000 déplacements). Le passage de l'estimation d'un nombre d'élèves et de travailleurs à celle du nombre de leurs déplacements est réalisé sur la base d'intensités hebdomadaires de déplacement, qui décroissent respectivement en fonction des populations citées (élèves : 4,5 et travailleurs : $4,21)$.

Tableau 2. Volumes des travailleurs liés à la RBC (le lieu de départ est défini par le lieu de départ déclaré) et de leurs déplacements selon le type de flux.

\begin{tabular}{|c|c|c|c|c|c|}
\hline & Travailleurs & $\begin{array}{l}\text { Part } \\
\text { dans le } \\
\text { total }\end{array}$ & $\begin{array}{l}\text { Fréquence } \\
\text { hebdomadaire de } \\
\text { jours avec un } \\
\text { déplacement }\end{array}$ & $\begin{array}{l}\text { Fréquence } \\
\text { hebdomadaire de } \\
\text { déplacement }^{\star}\end{array}$ & $\begin{array}{l}\text { Déplacements un jour } \\
\text { ouvrable }(\text { lu - ve })^{*}\end{array}$ \\
\hline & vol. & $\%$ & vol./semaine & vol./semaine & vol./jour ouvrable \\
\hline & A & B & C & $D=A \times C \times 2$ & $E=D \times 0,905^{* *} / 5$ \\
\hline $\begin{array}{l}\text { Travailleurs liés à } \\
\text { la RBC }\end{array}$ & 781705 & 100,0 & 4,16 & 6508000 & 1177000 \\
\hline Internes $(X)$ & 348752 & 44,6 & 4,13 & 2.880000 & 521000 \\
\hline Entrants $(Y)$ & 362979 & 46,4 & 4,19 & 3.040000 & 550000 \\
\hline Sortants $(Z)$ & 69974 & 9,0 & 4,20 & 588000 & 106000 \\
\hline $\begin{array}{l}\text { Travailleurs } \\
\text { bruxellois }(X+Z)\end{array}$ & 418726 & 53,6 & 4,14 & 3.469000 & 628000 \\
\hline $\begin{array}{l}\text { Travailleurs } \\
\text { employés en } \mathrm{RBC} \\
(\mathrm{X}+\mathrm{Y})\end{array}$ & 711730 & 91,0 & 4,16 & 5920000 & 1071000 \\
\hline
\end{tabular}

* Les résultats présentés sont arrondis au millier. **Selon Beldam 2010, 90,5 \% de l'ensemble des déplacements domicile-travail hebdomadaires ont lieu du lundi au vendredi inclus.

Sources : EFT 2011 - 2014, BELDAM 2010, calculs CES - USL-B 
Tableau 3. Nombre d'élèves (hors établissements internationaux), évolutions et volumes de leurs déplacements selon le type de flux.

\begin{tabular}{|c|c|c|c|c|c|}
\hline & Elèves & $\begin{array}{l}\text { Part dans le } \\
\text { total }\end{array}$ & $\begin{array}{c}\text { Fréquence } \\
\text { hebdomadaire de } \\
\text { jours avec un } \\
\text { déplacement }\end{array}$ & $\begin{array}{c}\text { Fréquence } \\
\text { hebdomadaire de } \\
\text { déplacement }^{\star}\end{array}$ & $\begin{array}{c}\text { Déplacements ur } \\
\text { jour ouvrable } \\
(\text { lu - ve })^{\star}\end{array}$ \\
\hline & vol. & $\%$ & vol./semaine & vol./semaine & vol./jour ouvrable \\
\hline & A & B & C & $D=A \times C \times 2$ & $E=D / 5$ \\
\hline RBC Élèves liés à la & 257138 & 100,0 & 4,5 & 2314000 & 463000 \\
\hline Internes $(X)$ & 212097 & 82,5 & 4,5 & 1909000 & 382000 \\
\hline Entrants (Y) & 35663 & 13,9 & 4,5 & 321000 & 64000 \\
\hline Sortants (Z) & 9378 & 3,6 & 4,5 & 84000 & 17000 \\
\hline $\begin{array}{l}\text { Elèves bruxellois } \\
(\mathrm{X}+\mathrm{Z})\end{array}$ & 221475 & 86,1 & 4,5 & 1993000 & 399000 \\
\hline $\begin{array}{l}\text { - Élèves } \\
\text { scolarisés en RBC } \\
(\mathrm{X}+\mathrm{Y})\end{array}$ & 247760 & 96,4 & 4,5 & 2230000 & 446000 \\
\hline
\end{tabular}

* Les résultats présentés sont arrondis au millier. Ce tableau ne reprend pas les étudiants de l'enseignement supérieur.

Sources : Communauté française et Vlaamse Gemeenschap 2014-2015, via IBSA

\section{Distances parcourues : déconnexion croissante entre lieu de résidence et lieu d'emploi/étude}

Les distances parcourues par les travailleurs dépendent fortement de leur lieu de résidence, mais également de leurs caractéristiques et statuts. Sans grande surprise, les travailleurs internes à la RBC parcourent des distances relativement courtes $(<5 \mathrm{~km}$ pour $70 \%$ des travailleurs), alors que les travailleurs entrants parcourent les distances en moyenne les plus longues ( $58,8 \%$ parcourent plus de $25 \mathrm{~km}$ ). Les travailleurs sortants tendent à occuper un travail en périphérie généralement assez proche de la RBC $(58,8 \%$ travaillent à moins de $25 \mathrm{~km}$ de leur lieu de domicile).

Plusieurs pays occidentaux ont pu observer une tendance à l'augmentation de la distance moyenne des déplacements domicile-travail (Dujardin et al., 2011). Ce constat vaut également pour la Belgique. Bien qu'elle soit moins nette pour ces dix dernières années (surtout pour la navette entrante), cette évolution résulte de la démocratisation de l'accès à la voiture individuelle et des gains de distance permis, au début, par l'augmentation de la vitesse automobile (Boussauw et al., 2009).

Nous pouvons, en effet, relier l'accroissement général des distances à la double contrainte de Zahavi, qui veut qu'une personne qui se déplace le ferait selon deux contraintes : une contrainte temporelle et une contrainte monétaire. L'accroissement de la vitesse permise par la motorisation a rendu possible l'augmentation des distances parcourues pour une même contrainte temporelle (Zahavi, 1974) mais cet accroissement de la vitesse n'est plus de mise aujourd'hui en raison de la saturation des réseaux de transport, mettant dès lors à mal la constante de Zahavi (Joly, 2005 ; van Wee et al., 2006). L'importance relative du lieu de travail dans le choix résidentiel des ménages a également évolué au fil du temps influant également sur les distances parcourues. En simplifiant, le fait de consacrer l'ensemble de sa carrière auprès d'un même employeur est devenu nettement moins 
courant aujourd'hui. Une des conséquences de ce changement peut être que les travailleurs seraient amenés à accepter des distances domicile-travail beaucoup plus importantes, plutôt que d'envisager une relocalisation de leur lieu de résidence plus proche de leur (nouvel) emploi, sachant que celui-ci ne sera peut-être pas durable. La croissance de la part des femmes sur le marché du travail participe aussi à l'augmentation du nombre de ménages dont les deux conjoints travaillent, complexifiant encore le choix de localisation résidentielle (Vanoutrive, 2012). La systématisation des remboursements sur les déplacements en train via l'introduction du système de tiers-payant à partir de 2004 (Boussauw \& Vanoutrive, 2017), de même que la fiscalité favorable aux voituressalaires, dont le nombre est en constante augmentation (May, 2017) peuvent également jouer un rôle sur l'allongement des distances domicile-travail.

La variation des distances peut également être considérée sous l'angle de la rareté ou de l'inadéquation de l'offre d'emploi à proximité. Sur le marché du travail, la spécialisation des activités professionnelles se décline en fonction de caractéristiques très variables, notamment selon le secteur d'activité, la taille de l'entreprise, le niveau de qualification ou le statut des travailleurs. Les fonctionnaires et les employés les plus qualifiés parcourent les distances les plus élevées en moyenne. C'est évidemment lié à la concentration à Bruxelles de nombreuses administrations internationales, fédérales ou des entités fédérées, ainsi que de nombreuses activités du tertiaire marchand recrutant une main-d'œuvre très qualifiée.

Certaines situations dessinent cependant un portrait plus nuancé. On constate, par exemple, que, parmi les Bruxellois, la part des travailleurs sortants est plus importante dans le «croissant pauvre» (au centre-ouest de la Région), depuis lequel les trajets sortants sont parcourus sur des distances en moyenne très élevées vers des lieux de travail répartis de manière singulièrement diffuse sur le territoire belge. La structure de l'emploi bruxellois, fortement marquée par le tertiaire très qualifié, est clairement défavorable aux travailleurs moins qualifiés, mais d'autres facteurs interviennent également (notamment les effets de « déqualification en cascade » dans le contexte d'un taux de chômage élevé (Devillé, 2008)) pour construire une situation, démontrée par ailleurs (Vandermotten, 2008), dans laquelle les travailleurs du croissant pauvre, à niveau de diplôme équivalent, auront également davantage de chances de se retrouver dans une situation de chômage. Le déficit d'emplois accessibles à ces Bruxellois semble être ici un facteur décisif imposant un surplus de mobilité vers la périphérie et au-delà alors que leur lieu de résidence est à proximité immédiate du plus grand pôle d'emploi du pays (Dewulf et al., 2015).

Tableau 4. Part des déplacements domicile-école (ou résidence-lieu d'étude) et domicile-travail selon la distance (à vol d'oiseau) et le type de flux (en \%).

\begin{tabular}{|c|c|c|c|c|c|c|c|}
\hline & $\begin{array}{ll}< & 5 \\
\mathrm{~km} & \end{array}$ & 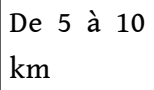 & $\begin{array}{l}\text { De } 10 \text { à } 15 \\
\mathrm{~km}\end{array}$ & $\begin{array}{l}\text { De } 15 \text { à } 25 \\
\mathrm{~km}\end{array}$ & $\begin{array}{l}\text { De } 25 \text { à } 50 \\
\mathrm{~km}\end{array}$ & $\begin{array}{l}> \\
\mathrm{km}\end{array}$ & Total \\
\hline $\begin{array}{l}\text { Actifs } \quad \text { occupés, } \\
\text { dont : }\end{array}$ & 25,8 & 16,5 & 7,9 & 12,9 & 20,6 & 16,4 & 100,0 \\
\hline $\begin{array}{l}\text { - déplacements } \\
\text { intérieurs }\end{array}$ & 70,0 & 28,3 & 1,7 & 0,0 & 0,0 & 0,0 & 100,0 \\
\hline
\end{tabular}




\begin{tabular}{|l|l|l|l|l|l|l|l|}
\hline - navetteurs entrants & 2,3 & 9,1 & 10,3 & 19,6 & 32,6 & 26,2 & 100,0 \\
\hline - navetteurs sortants & 4,9 & 17,9 & 16,3 & 19,7 & 23,5 & 17,6 & 100,0 \\
\hline Elèves, dont : & $\mathbf{8 3 , 2}$ & $\mathbf{1 0 , 4}$ & $\mathbf{2 , 4}$ & $\mathbf{1 , 9}$ & $\mathbf{1 , 3}$ & $\mathbf{0 , 8}$ & $\mathbf{1 0 0 , 0}$ \\
\hline $\begin{array}{l}\text { - déplacements } \\
\text { intérieurs }\end{array}$ & 92,9 & 6,8 & 0,3 & 0,0 & 0,0 & 0,0 & 100,0 \\
\hline - navetteurs entrants & 36,5 & 31,5 & 13,0 & 10,8 & 6,0 & 2,3 & 100,0 \\
\hline - navetteurs sortants & 0,1 & 4,4 & 11,5 & 24,0 & 27,0 & 33,1 & 100,0 \\
\hline CF) & 91,3 & 5,6 & 1,1 & 1,0 & 0,7 & 0,3 & 100,0 \\
\hline - niveau maternel & 88,2 & 7,4 & 1,7 & 1,4 & 0,9 & 0,4 & 100,0 \\
\hline - niveau primaire & 73,4 & 16,3 & 3,8 & 3,0 & 2,0 & 1,5 & 100,0 \\
\hline - niveau secondaire
\end{tabular}

Année 2011 pour les déplacements de travail et moyenne 2009-2014 pour les déplacements scolaires. Les écoles non reconnues par les Communautés françaises ou flamande ne sont pas prises en compte.

Sources : Communauté française, Vlaamse Gemeenschap 2009-2014 et Census 2011

Les déplacements domicile-école se caractérisent par des distances beaucoup plus courtes que les déplacements domicile-travail. Ainsi, plus de $80 \%$ des distances domicile-école sont inférieures à $5 \mathrm{~km}$ à vol d'oiseau (contre $26 \%$ des distances domicile-travail) même si les distances tendent à croître avec le niveau d'enseignement $(91,3 \%, 88,2 \%$ et $73,4 \%$ des distances domicile-école sont inférieures à $5 \mathrm{~km}$ respectivement pour les niveaux maternel, primaire et secondaire). Pour autant, la mobilité liée à l'école dépasse largement l'échelle strictement locale puisque plus d'un élève sur deux (54\%) parcourt au moins $1 \mathrm{~km}$ pour rejoindre son école et un sur trois (32\%) parcourt au moins $2 \mathrm{~km}$.

Dans le détail, les distances domicile-école résultent d'un jeu complexe de facteurs. Elles dépendent bien sûr en partie du maillage plus ou moins serré des implantations scolaires et de leur accessibilité en transports en commun. Le niveau scolaire importe également: on constate une élévation des distances parcourues avec la montée en âge, qui peut se lire par une capacité croissante des individus à se déplacer (autonomie vis-à-vis des parents, acquisition de compétences d'orientation dans les territoires et d'usage des réseaux de transport public, passage du permis de conduire, accès à une voiture, etc.) qui relâchent progressivement la contrainte de proximité entre le lieu de domicile et l'activité scolaire.

Elle peut également s'interpréter par une segmentation accrue de l'offre scolaire, en même temps qu'un approfondissement de la spécialisation des trajectoires individuelles : l'offre scolaire ou professionnelle pertinente à chacun devient généralement de plus en plus spécifique et rare à mesure que l'on avance dans son parcours de formation. Elle impose de ce fait, en moyenne, de parcourir des distances de plus en plus importantes.

En ce qui concerne les déplacements domicile-école, l'offre se structure en fonction des types d'enseignement (ordinaire ou spécialisé), des filières (à partir du secondaire: général, technique, professionnel), de la langue (enseignement francophone ou 
néerlandophone) mais aussi de la réputation de l'établissement. Il résulte de cette grande diversité une couverture spatiale du territoire bruxellois à la fois segmentée et très inégale au sein de chacun de ces segments. Cette situation génère un surplus de distances à parcourir par rapport à une offre qui serait idéalement moins segmentée et plus également répartie. Ainsi, dès le fondamental, les mobilités entre domicile et école sont loin d'être restreintes à l'échelle du quartier. Ces surplus de déplacements concernent par ailleurs au premier chef les individus plus "vulnérables » : élèves dans l'enseignement spécialisé, élèves en retard scolaire à partir du deuxième degré du secondaire, élèves en ascension sociale, etc. Enfin, la surimposition de la croissance démographique à un tel contexte a pour effet une élévation globale des distances sur les trajets intra-régionaux (les parents sont forcés d'accepter des distances plus longues dans le contexte d'une offre à la fois fragmentée spatialement et saturée), dont l'impact est plus fortement marqué dans le fondamental, qui a absorbé le premier les effets de la croissance.

\section{Evolution modale} nombreuses variables influencent le choix modal (facteurs personnels, liés au mode ou liés au déplacement) (De Witte, 2012, pp. 123-142). En ce qui concerne les travailleurs liés à la $\mathrm{RBC}$, chaque mode parait compétitif sur certaines distances bien précises. Ainsi, la marche est logiquement restreinte aux distances très courtes. Les métros, trams et bus sont particulièrement utilisés sur les distances inférieures à $15 \mathrm{~km}$ et le train pour les distances longues (généralement au-delà de $50 \mathrm{~km}$ ). L'automobile est caractérisée par un usage important quelle que soit la distance même si, dans le contexte des déplacements liés à la RBC, elle domine plus spécifiquement entre 15 et $40 \mathrm{~km}$. Cette distribution modale selon la distance souligne en creux l'influence de l'accessibilité en transport en commun, tant à l'origine qu'à destination, sur les choix modaux (par la présence d'une alternative en transport public valable en milieu urbain et son absence depuis la proche périphérie), qui représente un levier évident du report modal (voir Vanoutrive et al., 2011, 2012). L'amélioration de la desserte en train (Réseau S) peut toutefois conduire également à une augmentation des distances parcourues (Boon \& Gayda, 2000).

En ce qui concerne l'ensemble des travailleurs liés à la RBC, la moitié $(49,7 \%)$ utilise la voiture pour se rendre sur leur lieu de travail. Le deuxième mode de transport le plus utilisé est le train, avec une part de $21,7 \%$, suivi des transports publics urbains (métro, tram, bus) avec 20,6\%. Les transports en commun sont le premier mode de déplacement des travailleurs internes $(42,4 \%)$, favorisé par un réseau beaucoup plus accessible aussi bien à l'origine qu'à destination. La voiture est reléguée à la deuxième position, avec une part à hauteur de $37,5 \%$, ce qui demeure très élevé au regard de l'offre de transports publics disponible et de la proximité des lieux de travail. Parmi les travailleurs entrants, la voiture reste le premier mode de déplacement ( $49,6 \%$ des travailleurs) mais le train y est le plus spécifique $(41,6 \%$ ) en raison à la fois des distances parcourues très importantes et de la bonne accessibilité en train des lieux de travail. Enfin, les travailleurs sortants sont davantage dépendants du mode automobile qu'ils utilisent à hauteur de 73,3\%, ce qui s'explique pour partie par le manque de transports en commun à destination. 
Tableau 5. Mode de déplacement principal des travailleurs liés à la RBC.

\begin{tabular}{|c|c|c|c|c|c|c|c|c|c|c|c|}
\hline & \multicolumn{8}{|c|}{ Mode de déplacement principal } & \multirow[b]{2}{*}{$\begin{array}{c}\text { Auto } \\
\text { (pass.) }\end{array}$} & \multirow[b]{2}{*}{ Travailleurs } & \multirow[b]{2}{*}{ Répondants } \\
\hline & Marche & Vélo & $\begin{array}{l}\text { Moto / } \\
\text { scooter }\end{array}$ & MTB & Train & $\begin{array}{c}\text { Auto } \\
\text { (passager) }\end{array}$ & $\begin{array}{c}\text { Auto } \\
\text { (conducteur) }\end{array}$ & $\begin{array}{c}\text { Auto } \\
\text { (pass.) } \\
\text { / Auto } \\
\text { (total) }\end{array}$ & & & \\
\hline & $\%$ & $\%$ & $\%$ & $\%$ & $\%$ & $\%$ & $\%$ & $\%$ & $\%$ & vol. & vol. \\
\hline $\begin{array}{r}\text { Travailleur } \\
s \text { liés à la RBC }\end{array}$ & 5,3 & 1,8 & 1,1 & 20,6 & 21,7 & 3,7 & 45,9 & 49,7 & 8,1 & 642.043 & 25.699 \\
\hline \multicolumn{12}{|c|}{ Flux de travailleurs } \\
\hline Internes & 11,7 & 3,2 & 1,3 & 40,5 & 1,9 & 3,8 & 37,5 & 41,4 & 10,2 & 278.644 & 12.042 \\
\hline Entrants & 0,3 & 0,7 & 0,9 & 3,8 & 41,6 & 3,1 & 49,6 & 52,7 & 6,3 & 304.890 & 11.191 \\
\hline Sortants & 0,6 & 0,7 & 0,8 & 13,0 & 11,7 & 6,5 & 66,8 & 73,3 & 9,7 & 58.509 & 2.466 \\
\hline \multicolumn{12}{|l|}{ Distances $^{*}$} \\
\hline$<5 \mathrm{~km}$ & 26,2 & 4,8 & 1,2 & 33,9 & 0,7 & 3,3 & 29,9 & 33,2 & 11,1 & 120.618 & 5.173 \\
\hline $5-10 \mathrm{~km}$ & 0,9 & 2,6 & 1,3 & 44,6 & 1,6 & 4,0 & 44,9 & 48,9 & 8,8 & 122.001 & 5.187 \\
\hline $10-15 \mathrm{~km}$ & 0,3 & 1,5 & 1,8 & 29,0 & 4,9 & 4,4 & 58,1 & 62,5 & 7,6 & 66.210 & 2.720 \\
\hline $15-25 \mathrm{~km}$ & 0,1 & 0,9 & 1,2 & 13,9 & 17,6 & 4,3 & 62,0 & 66,4 & 7,0 & 76.560 & 3.112 \\
\hline $25-50 \mathrm{~km}$ & 0,0 & 0,5 & 0,9 & 2,7 & 42,4 & 3,4 & 50,2 & 53,5 & 6,7 & 128.249 & 4.883 \\
\hline$>50 \mathrm{~km}$ & 0,2 & 0,0 & 0,4 & 1,1 & 51,9 & 3,6 & 42,8 & 46,4 & 8,5 & 124.510 & 4.435 \\
\hline
\end{tabular}

*distances parcourues entre le lieu de domicile et le lieu de travail déclarées par les répondants.

Source : EFT 2011-2014

En termes d'évolution des modes de déplacement, on observe un recul de la voiture au profit du transport public (bus, tram, métro et train), tous flux confondus mais davantage parmi les internes, pour lesquels l'alternative en transport public est certainement la plus dense. Ceci se produit dans un contexte où les freins à l'usage de la voiture se multiplient (baisse du niveau socio-économique, élévation de la congestion automobile, réduction des facilités de stationnement, réduction de l'espace public dévolu à l'automobile, changement d'attitude envers la possession automobile, etc.).

Nous constatons également une diminution de la part des passagers en voiture parmi les travailleurs entrants alors qu'elle est en progrès parmi les travailleurs internes et surtout sortants. Le contexte d'élévation globale des distances qui va de pair avec une dispersion à la fois des lieux de domicile et des lieux de travail joue ici un rôle, comme en témoigne la part plus importante de passagers parmi les automobilistes internes à la Région et le recul de l'indicateur avec la distance. Ceci explique au passage la difficulté de mettre en œuvre des pratiques de covoiturage efficaces au niveau des entreprises dans le contexte de lieux de résidence éclatés. Cependant, il ne faut pas surévaluer le déterminisme spatial et il semble que la disponibilité moindre d'un véhicule (ouvriers, travailleurs faiblement qualifiés) ou les logiques sectorielles (secteur de la construction) puissent former les conditions d'émergence d'une pratique soutenue du covoiturage.

Par rapport aux déplacements domicile-travail, les trajets liés à l'école s'inscrivent dans une double spécificité : celle de l'âge (et de l'autonomie liée à celui-ci) et celle de la plus grande fréquence de trajets courts. Ces deux facteurs influent directement sur le mode de transport utilisé. Globalement, tous niveaux, types et Communautés confondus, les trajets domicile-école sont caractérisés par une part modale importante de la marche et des 
transports en commun. Les parts modales varient cependant considérablement selon les niveaux.

Dans le fondamental, où les élèves ont moins d'autonomie, la part des transports publics est notablement plus réduite, avec seulement $18 \%$, tandis que la part des élèves conduits en voiture s'élève à $40 \%$. Dans le cadre de trajets domicile-école, souvent courts, la marche représente, avec $36 \%$, le deuxième mode principal. Dans le secondaire, le transport public domine fortement: il s'agit du mode principal dans un peu plus de la moitié des cas (57\% pour l'enseignement francophone, et $62 \%$ pour le néerlandophone). On constate en revanche des parts beaucoup plus faibles aussi bien de la marche (dans le cadre de trajets souvent plus longs) que de la voiture qui se partagent à peu près équitablement la deuxième position.

Figure 3. Part des modes de déplacement dans l'enseignement obligatoire : mode principal (selon la plus grande distance).

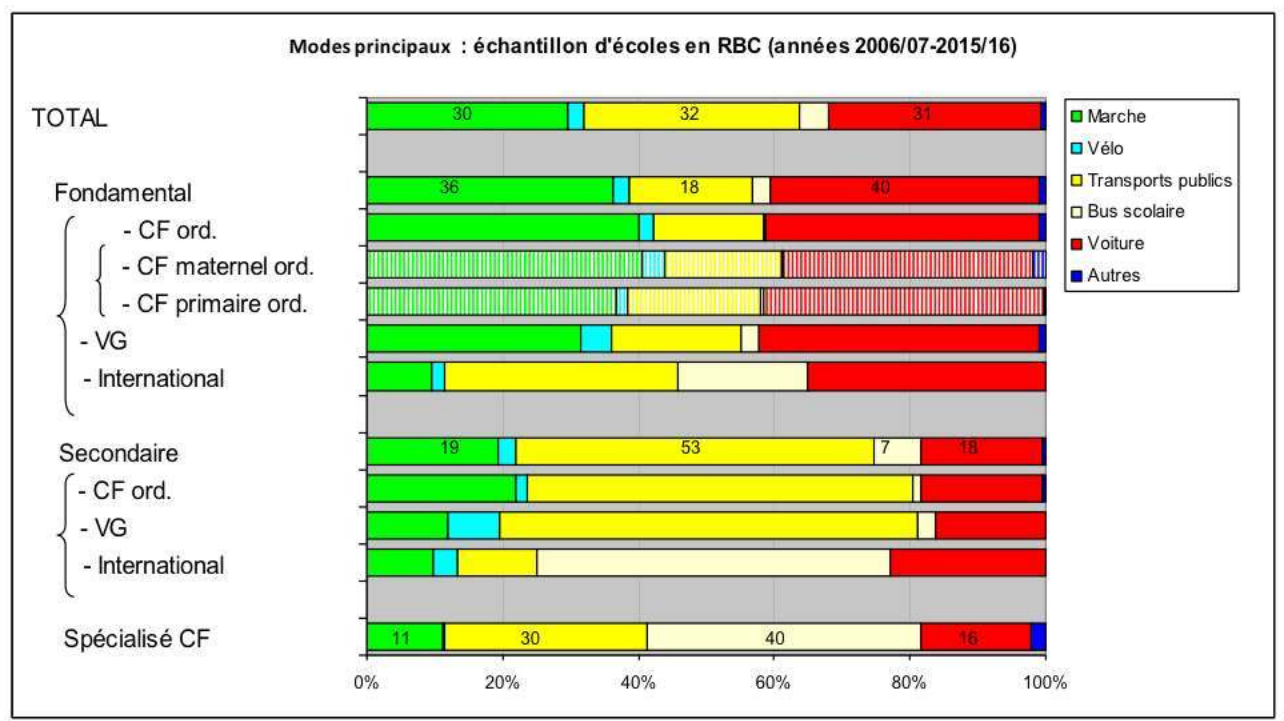

Remarques: (1) Ces données incorporent les écoles internationales et (2) les données relatives aux écoles maternelles, reprises en hachuré, doivent être lues avec beaucoup de précaution : elles ne reposent que sur une partie des écoles fondamentales.

Sources : Plans de déplacements scolaires, années variables de 2006 à 2014, et prédiagnostics scolaires 2014

\section{Conclusions et perspectives}

\section{Des constats}

Avec l'abandon des recensements de la population, l'analyse des DDT et des DDE constitue un challenge méthodologique que seul le croisement de plusieurs sources permet de rencontrer, de manière parfois lacunaire, comme c'est le cas par exemple pour les étudiants de l'enseignement supérieur.

Un certain nombre de constats se dégagent néanmoins de l'analyse. Comme nous l'avons vu, les DDT et DDE, même minoritaires dans le total des déplacements, n'en restent pas moins très structurants pour la mobilité quotidienne, en particulier les jours ouvrables. Ce sont plus de 1.640.000 déplacements quotidiens (sans compter ceux des étudiants du 
supérieur) qui, en moyenne, sont générés dans le cadre des DDT ou des DDE en lien avec la $\mathrm{RBC}$, une ville d'à peine 1,2 million d'habitants.

L'observation que nous avons opérée des heures de départ les jours ouvrables donne à penser que la pénibilité de ces déplacements s'aggrave, vu notamment leur horaire de plus en plus matinal, mais aussi suite à l'augmentation de la durée des trajets que ce soit en raison de la congestion des infrastructures de transports ou de l'allongement des distances parcourues, dû à de multiples causes que nous avons examinées. À Bruxelles comme ailleurs, la fameuse "constante de Zahavi» est remise en cause : l'allongement des distances parcourues n'est plus compensé par des gains de vitesse. Et ce phénomène risque d'encore s'accentuer à l'avenir dans le contexte de croissance démographique que connait l'aire métropolitaine bruxelloise.

L'augmentation de la navette sortante pour les DDT des Bruxellois est un autre constat que nous avons fait de manière différenciée pour les différentes catégories de travailleurs. En particulier, ceux issus du « croissant pauvre » bruxellois sont ainsi de plus en plus contraints, pour diverses raisons que nous avons analysées, de parcourir des distances en moyenne très élevées vers des lieux de travail répartis de manière singulièrement diffuse sur le territoire belge.

Sans surprise, les déplacements domicile-école se caractérisent quant à eux par des distances beaucoup plus courtes que les déplacements domicile-travail. On constate néanmoins une élévation des distances parcourues avec la montée en âge, qui peut se lire par une capacité croissante à se déplacer qui relâche progressivement la contrainte de proximité entre le lieu de domicile et l'activité scolaire. Mais cela reflète également la segmentation accrue de l'offre scolaire bruxelloise, en même temps qu'un approfondissement de la spécialisation des trajectoires individuelles

Enfin, nous avons montré que distances parcourues et pratiques de déplacements étaient intimement liées dans la détermination du choix du mode de transport, même si de nombreuses autres variables influencent celui-ci également. C'est ainsi que les modes durables (marche, vélo, transports publics) sont davantage utilisés pour les DDT internes à la $\mathrm{RBC}$ que pour les déplacements entrants et sortants. De même, en termes d'évolution des modes de déplacement, nous avons observé un recul de la voiture au profit du transport public (bus, tram, métro et train), tous flux confondus mais davantage parmi les internes, pour lesquels l'alternative en transport public est certainement la plus dense. Nous avons toutefois mis le lecteur en garde contre toute forme de déterminisme spatial, comme dans le cas de la diminution de la part des passagers en voiture parmi les travailleurs entrants, alors qu'elle est en progrès parmi les travailleurs internes et surtout sortants. D'autres phénomènes comme la moindre disponibilité d'un véhicule (ouvriers, travailleurs faiblement qualifiés) ou des logiques sectorielles (secteur de la construction) peuvent en effet expliquer l'émergence d'une pratique soutenue du covoiturage.

Pour ce qui est des DDE, les parts modales varient considérablement selon les niveaux et la distance entre le domicile et le lieu de scolarisation.

\section{Des pistes d'action}

41 La congestion automobile met sous tension les routines de déplacement. Plusieurs études qualitatives tendent en effet à montrer que le comportement modal est déterminé par la ponctualité et la fiabilité d'un mode de transport. Il apparaît également, lors de simulations (Bates et al., 2001), que les usagers tendent à donner davantage d'importance 
à une réduction de la variabilité qu'à la durée moyenne d'un déplacement. En d'autres termes, l'on préférera un mode fiable, quitte à prendre plus de temps, à un mode moins fiable qui permettrait un déplacement moins long.

La congestion automobile touche directement à cet aspect. D'une part, elle entraîne une baisse des vitesses de déplacement et, d'autre part, elle augmente la variabilité des temps de parcours. L'inertie des routines est pourtant importante. Des études ont tenté de mesurer le seuil de tolérance des ménages à la congestion automobile. De l'une d'elles, menée en France en 2010 (enquête de préférences déclarées), il est ressorti que les individus et les ménages tentent, autant que possible, de préserver leur programme d'activités, n'acceptant que des ajustements marginaux (partir plus tôt, changer d'itinéraire) n'affectant pas considérablement leurs routines, en particulier en cas de déplacements fortement contraints (par exemple, un déplacement domicile-travail, par opposition à une activité récréative non obligatoire). Le seuil de tolérance à la congestion tournerait autour de trente minutes supplémentaires, en deçà duquel les individus considèrent pouvoir préserver leurs routines grâce à des ajustements marginaux. Audelà, ils tendraient à modifier leur comportement (utiliser un autre mode ou chaîner les déplacements, réduire la fréquence ou l'horaire de l'activité, déménager ou annuler son activité...). Ce seuil tendrait à augmenter pour les personnes utilisant principalement la voiture et habitant en périphérie, par rapport à des personnes aux déplacements multimodaux habitant en zone urbaine (Lesteven, 2015).

Une piste souvent évoquée pour réduire le nombre de déplacements hebdomadaires, et par-là, influer sur la congestion, est le télétravail. L'encouragement du télétravail, mentionné dans les Plans de déplacement d'entreprise (PDE) semble connaitre un succès certain auprès des travailleurs des entreprises bruxelloises soumis à l'obligation PDE ( $16 \%$ des travailleurs de ces entreprises pratiquaient le télétravail en 2014 et prenaient en moyenne un jour de télétravail par semaine). Au niveau individuel, le recours au télétravail à la maison peut représenter une "soupape » dans la semaine en termes de routines, le temps non passé à se déplacer pouvant être réinvesti dans d'autres tâches (qui, comme les courses par exemple, peuvent générer à leur tour des déplacements). Soulignons cependant que le télétravail n'est pas une mesure applicable à l'ensemble des travailleurs et qu'il est restreint aux activités de bureau. De ce point de vue, il est intéressant de constater qu'il concerne davantage les travailleurs entrants, qui sont ceux qui parcourent en moyenne les distances les plus élevées. Les employés moins qualifiés et les ouvriers (davantage représentés parmi les internes et les sortants), pour lesquels l'exigence de présence sur le lieu de travail est plus forte, sont, sans surprise, moins représentés.

La flexibilisation des horaires de travail peut également contribuer à l'étalement dans le temps des déplacements domicile-travail par une plus grande flexibilité dans le choix des heures d'arrivée et départ des travailleurs. Actuellement, les horaires flottants concernent $12 \%$ des travailleurs. Mais cette flexibilisation peut aussi entraîner la croissance des horaires atypiques et horaires flexibles imposés, surtout parmi les travailleurs les moins qualifiés ( $16 \%$ des ouvriers, $13 \%$ des travailleurs ayant au plus un diplôme du secondaire) et plus encore dans certains secteurs d'activité particuliers: l'horeca ( $22 \%)$, le transport et l'entreposage ( $21 \%)$, les soins de santé publique et l'action sociale $(18 \%)$, le commerce, la réparation de véhicules $(13 \%)$ ou encore les activités de soutien aux entreprises (12,5\%) (EFT 2011-2014). Les travailleurs à temps partiel, qui sont 
majoritairement des femmes, tendent également à se déplacer davantage, comparativement aux travailleurs à équivalent temps plein.

La flexibilisation du travail peut donc contribuer à la production de contraintes lourdes sur l'élaboration des routines quotidiennes des travailleurs peu qualifiés (dont l'accès à la voiture est plus faible) et des femmes (qui devront en moyenne cumuler ces tensions avec davantage de tâches annexes). Dans le même ordre d'idées, la question de la fixité du lieu de travail est absente des enquêtes ( $7 \%$ des travailleurs belges ont un lieu de travail variable selon Beldam 2010), alors qu'il s'agit d'une dimension importante du point de vue de la stabilité des routines quotidiennes.

Une réponse simple mais qui va à l'encontre des tendances actuelles serait de limiter, voire de réduire, cette flexibilisation décrite de l'emploi. En l'absence d'un tel renversement, cette problématique appelle une réponse en termes d'action publique sur la mobilité qui s'éloigne des objectifs classiques de report modal et de réduction de la congestion, et qui peut même les contredire sur certains aspects. Il s'agirait en effet de renforcer la "motilité » (Kaufmann, 2001 et 2002 ; Kaufmann et al, 2015) des individus, c'est-à-dire de les doter d'outils et de moyens (cours de conduite, accès à un véhicule automobile, tarifs préférentiels pour le transport public, apprentissage de l'intermodalité et de la multimodalité, support par des "coachs mobilité », vélo-école, etc.) leur permettant d'avoir une plus grande aisance et une plus grande adaptabilité dans le cadre des exigences de déplacement qu'ils rencontrent au quotidien.

L'analyse met également en évidence que, pour lutter contre l'allongement des distances, une action sur l'offre d'activités quotidiennes peut s'avérer pertinente. Favoriser la création d'emplois peu qualifiés en $\mathrm{RBC}$, au-delà de son effet évident sur la mise à l'emploi des Bruxellois, permettrait de pallier, dans une certaine mesure, les surplus de distances qui incombent aux travailleurs de certains quartiers (issus du croissant pauvre en particulier). Au-delà de l'élargissement global de l'offre, il s'agirait d'améliorer la couverture spatiale, relativement aux besoins de la population, des différents niveaux de l'offre (Boussauw et al., 2012 ; Boussauw \& Vanoutrive, 2017), ou de limiter le degré de segmentation de celle-ci. Au niveau scolaire, cela peut signifier de viser une certaine homogénéisation de l'attractivité des établissements, aujourd'hui très fortement hiérarchisés en termes de réputation et de niveau socio-économique, mais encore de réduire la segmentation de l'enseignement lui-même, en promouvant par exemple la diversité des enseignements dans les programmes ou en élargissant les possibilités de retour vers le général.

Complémentairement, une planification territoriale favorisant les implantations résidentielles, scolaires et de pôles d'emplois autour des nœuds de transports publics aurait pour effet de rendre plus accessible l'offre pertinente à chacun, particulièrement pour les personnes qui ne disposent pas d'un accès aisé à l'automobile (voir aussi Boussauw \& Vanoutrive, 2017). Accessoirement, en renforçant l'attractivité des transports en commun, ce type de planification est évidemment de nature à favoriser le report modal vers ceux-ci et à lutter contre la congestion automobile. 


\section{BIBLIOGRAPHIE}

AUJEAN L., CASTIAU E., ROELANDTS M., VANDERMOTTEN C. (2007), « Le positionnement des villes belges dans le réseau global des services avancés », Belgeo, 8, 1, pp. 15-29.

BASTIN G. (2013), Plans de déplacements d'entreprise - Bilan de la situation 2011, Bruxelles Environnement, Bruxelles Mobilité.

BATES J., POLAK J., JONES P., COOK A. (2001), « The valuation of reliability for personal travel », Transportation Research, Part E, 37, pp. 191-229.

BBP-ADT-ATO (2012), Analyse de l'adéquation entre l'offre et les besoins en places scolaires dans l'enseignement primaire, décembre 2012, http://www.adt-ato.brussels/sites/default/files/ documents/Rapport_primaire_complet-fr.pdf.

BBP-ADT-ATO (2012), Analyse de l'adéquation entre l'offre et les besoins en places scolaires dans l'enseignement maternel, décembre 2012, http://www.adt-ato.brussels/sites/default/files/ documents/Rapport_maternel\%20_complet-fr.pdf.

BBP-ADT-ATO (2012), Analyse de l'adéquation entre l'offre et les besoins en places scolaires dans l'enseignement secondaire, décembre 2012, http://www.adt-ato.brussels/sites/default/files/ documents/Rapport_secondaire_complet-fr_0.pdf.

BOON F., GAYDA S. (2000), « Quelques impacts du futur RER sur la Région bruxelloise et sa périphérie », STRATEC s.a., Communication au $14^{\mathrm{e}}$ Congrès des économistes belges de langue français, http://www.stratec.be/sites/default/files/files/IMPACTSRER2-FB.pdf.

BOUSSAUW K., VANOUTRIVE T. (2017), « Transport policy in Belgium: Translating sustainability discourses into unsustainable outcomes ", Transport Policy, 53, p. 1119.

BOUSSAUW K., NEUTENS T. \& WITLOX F. (2012), « Relationship between Spatial Proximity and Travel-to-Work Distance: The Effect of the Compact City », Regional Studies, 46, 6, 687706.

BOUSSAUW K., WITLOX F. (2009), « Introducing a commute-energy performance index for Flanders ", Transportation Research Part A: Policy and Practice, 43, 5, 580591.

BRUXELLES ENVIRONNEMENT (2016), Les plans de déplacements d'entreprise en Région de BruxellesCapitale. Bilan de la situation 2014 , Bruxelles.

COLARD A., MARISSAL P., VANDERMOTTEN C. \& VAN HAMME G. (1997), Emploi et structures socioéconomiques régionales, Recensement Général de la Population et des Logements au 1er mars 1991, Monographie, $\mathrm{n}^{\circ}$ 6, Bruxelles.

COMMENGES H. (2013), L'invention de la mobilité quotidienne. Aspects performatifs des instruments de la socio-économie des transports, thèse de géographie, Université Paris-Diderot - Paris VII.

COMMENGES H. (2015), « Mesurer les pratiques modales et la dépendance à l'automobile : à la recherche de la congruence entre mesure et interprétation », Espace populations sociétés [En ligne], 2015/1-2, mis en ligne le 01 juillet 2015, consulté le 03 septembre 2015, http:// eps.revues.org/5935.

CORNELIS E., et al. (2012), Belgian Daily Mobility 2012 (BELDAM). Rapport de recherche, naXys (Université de Namur), IMOB (Université d'Hasselt), CES (Université Saint-Louis - Bruxelles). 
DEVILLÉ H. (2008), « Le chômage bruxellois entre inadéquation de qualification et déqualification en cascade ", Brussels Studies, [En ligne], Collection générale, $n^{\circ} 14$, mis en ligne le 21 janvier 2008, consulté le 02 novembre 2017, http://brussels.revues.org/523.

DEWULF N., BOUSSAUW K., BOELENS L., RYCKEWAERT M. (2015), The spatial mismatch hypothesis in Brussels and its fringe: implications for transport and urban development, $29^{\text {th }}$ International conference of the Association of European Schools of Planning: Definite Space - Fuzzy Responsibility, 13-16 July 2015, https://biblio.ugent.be/publication/6884046/file/6884053.

DICKINSON R.E. (1957), « The geography of commuting : the Netherlands and Belgium », Geographical Review, 47, 4.

DUJARDIN S., BOUSSAUW K., BREVERS F., LAMBOTTE J.-M., TELLER J. \& WITLOX F. (2011), Hometo-work commuting, spatial structure and energy consumption : a comparative analysis of Wallonia and Flanders, Belgium, presentation paper, BIVEC/GIBET Transport Research Day, pp. 97-110.

ERMANS T., BRANDELEER C., D'ANDRIMONT C., HUBERT M., MARISSAL P., VANDERMOTTEN C., WAYENS B. (à paraître), Analyse des déplacements domicile-travail et domicile école en $R B C$, Cahier de l'observatoire de la mobilité de la RBC, 6.

HERMIA J.-P. (2016), Baromètre démographique 2016 de la Région de Bruxelles-Capitale, Focus de l'IBSA, décembre 2016 [En ligne], http://ibsa.brussels/fichiers/publications/focus-de-libsa/

focus_16_decembre_2016.

HUBERT M., LEBRUN K., HUYNEN P. \& DOBRUSZKES F., (2013) « Note de synthèse BSI. La mobilité quotidienne à Bruxelles : défis, outils et chantiers prioritaires ", Brussels Studies [En ligne], Notes de synthèse, $\mathrm{n}^{\circ} 71$, mis en ligne le 18 septembre 2013, consulté le 02 novembre 2017. http:// brussels.revues.org/1184.

INSTITUT NATIONAL DE STATISTIQUE (1989), Recensement de la Population et du Logement 1981, Tome 9, Mobilité géographique de la main-d'œuvre, Bruxelles.

JOLY I. (2005), "Décomposition de l'hypothèse de constance des budgets-temps de transport”, in MONTULET B., HUBERT M., JEMELIN C. \& SCHMITZ S. (éd.), in Mobilités et temporalités, Bruxelles, Publications des Facultés universitaires Saint-Louis, Travaux et recherches 51, pp. 129-150.

JUAN S. (dir.), LARGO-POIRIER A., ORAIN H. \& POLTORAK J.F. (1997), Les sentiers du quotidien : rigidité, fluidité des espaces sociaux et trajets routiniers en ville, Paris, L'Harmattan, collection Villes et entreprises.

JUAN S. (2015), « Le concept de routine dans la socio-anthropologie de la vie quotidienne », Espace populations sociétés [En ligne], 2015/1-2, mis en ligne le 01 juillet 2015, consulté le 03 septembre 2015, http://eps.revues.org/5935.

KAUFMANN V., (2001), “La motilité : une notion clé pour revisiter l'urbain”, in BASSAND M., KAUFMANN V., \& JOYE D. (éd.), Enjeux de la sociologie urbaine, Lausanne, Les Presses polytechniques et universitaires romandes, Science, technique, société, pp. 87-102.

KAUFMANN V. (2002), Re-Thinking mobiliy. Contemporary Sociology, Ashgate, Aldershot, UK.

KAUFMANN V., RAVALET E. \& DUPUIS E. (éd.) (2015), Motilité et mobilité : mode d'emploi, Neuchâtel, Editions Alphil - Presses universitaires suisses, 248 p.

LEBRUN K., HUBERT M., HUYNEN P., DE WITTE A. \& MACHARIS C. (2013), Les pratiques de déplacement à Bruxelles, Cahiers de l'Observatoire de la mobilité de la Région de Bruxelles-Capitale, 2. 
LEBRUN K., HUBERT M., HUYNEN P. \& PATRIARCHE G. (2014), Les pratiques de déplacement à Bruxelles : analyses approfondies, Cahiers de l'Observatoire de la mobilité de la Région de BruxellesCapitale, 3.

LESTEVEN G. (2015), « La congestion automobile perturbe-t-elle la routine des ménages motorisés ? Illustration à partir de l'Ile-de-France ", Espace populations sociétés [En ligne], 2015/1-2, mis en ligne le 01 juillet 2015, consulté le 03 septembre 2015, http:// eps.revues.org/5935.

MAY X. (2017) «L'épineuse question du nombre de voitures de société en Belgique », Brussels Studies [En ligne], Fact Sheets, $\mathrm{n}^{\circ} 113$, mis en ligne le 10 juillet 2017, consulté le 01 novembre 2017, https://journals.openedition.org/brussels/1533.

MARISSAL P., MEDINA LOCKHART P., VAN HAMME G., KESTELOOT C. \& VANDERMOTTEN C. (2008), Les structures socio-économiques de l'espace belge. Une exploitation des données d'emploi de l'enquête socio-économique de 2001, Monographie ${ }^{\circ} 6$, SPF Economie, Politique scientifique fédérale.

MEISSONIER J., RICHER C. (2015), « Métro-boulot-dodo : quoi de neuf dans nos routines de mobilité ? », Espace populations sociétés [En ligne], 2015/1-2, mis en ligne le 01 juillet 2015, consulté le 03 septembre 2015, http:// eps.revues.org/5935.

MÉRENNE-SCHOUMAKER B., VAN DER HAEGEN H., VAN HECKE E., HALLEUX J.-M., JUCHTMANS G. \& DERWAEL J.M. (1999), Recensement Général de la Population et du Logement 1991, Monographie 11b, Migrations de travail et migrations scolaires », Bruxelles, Institut National de Statistique.

MONTULET B., HUBERT M. \& HUYNEN P., 2007, Etre Mobile. Vécus du temps et usages des modes de transport à Bruxelles, Bruxelles, Publications des Facultés universitaires Saint-Louis, 208 p.

MONTULET B., HUBERT M. (2008), « Se déplacer à Bruxelles avec des enfants ? Une étude sociologique sur les vécus du temps et les usages des modes de transport », Brussels Studies [En ligne], Collection générale, 15, mis en ligne le 11 février 2008, consulté le 12 janvier 2017, http:// brussels.revues.org/535.

PAUWELS C., ANDRIES P. (2015), Diagnostics des déplacements domicile - lieu de travail 2014, Bruxelles, Service Public Fédéral Mobilité et Transports.

PRADEL B., CHARDONNEL S., CAILLY L. \& FOURNY M.C (2015), « Les routines de déplacement dans les espaces périurbains : les dimensions collectives des agencements quotidiens », Espace populations sociétés, [En ligne], 2015/1-2, mis en ligne le 01 juillet 2015, consulté le 05 novembre 2015, http://eps.revues.org/5961.

STATISTICS BELGIUM (s.d.), Census 2011 : Déplacements domicile lieu de travail, http:// census2011.be/analyse/flux_fr.html, consulté le 29/6/2017.

VAESEN J., WAYENS B., avec la collaboration de VAN HEUR B., YSEBAERT W., D'ANDRIMONT C., KESBEKE W., HUBERT M., JAUMAIN M., AMEZ L., CORIJN E., DE LAET S., DOB- BELS J., DOTTI N. F., JOURDAIN V., TAYMANS M. \& VAN DROOGENBROECK N., (2014), « Note de synthèse BSI.

L'enseignement supérieur et Bruxelles ", Brussels Studies [En ligne], Notes de synthèse, $\mathrm{n}^{\circ} 76$, mis en ligne le 23 avril 2014, consulté le 02 novembre 2017, http://brussels.revues.org/1214.

VAN DER HAEGHEN H. (1953), « Les migrants alternants bruxellois », Bulletin de la Soc. Belge d'Etudes géographiques, 22, pp. 441-449.

VAN WEE B., RIETVELD P. \& MEURS H. (2006), « Is average daily travel time expenditure constant? In search of explanations for an increase in average travel time », Journal of Transport Geography, 14, pp. 109-122. 
VANDERMOTTEN C. (2008), Évolution socio-économique, reproduction sociale et formation à Bruxelles, Bruxelles, Commission Consultative Formation Emploi Enseignement en Région de BruxellesCapitale-CCFEE.

VANDERMOTTEN C. (2014), Bruxelles, une lecture de la ville, Bruxelles, Éditions de l'Université de Bruxelles, Collection UBLire.

VANOUTRIVE T., VAN MALDEREN L., JOURQUIN B., THOMAS I., VERHETSEL A. \& WITLOX F. (2011), « Rail commuting to workplaces in Belgium : a multilevel approach », International journal of sustainable transportation, 6, pp. 67-87.

VANOUTRIVE T., VAN DE VIJVER E., VAN MALDEREN L., JOURQUIN B., THOMAS I., VERHETSEL I. \& WITLOX F. (2012), « What determines carpooling to workplaces in Belgium : location, organisation or promotion? ", Journal of transport geography, 22, pp. 77-86.

VANOUTRIVE T., (2012), « Scale and the workplace as level of analysis in transport geography », Belgeo, 1-2, pp. 1-19, http://belgeo.revues.org/6229.

VERHETSEL A., VAN HECKE E., THOMAS I., BEELEN M., HALLEUX J.-M., LAMBOTTE J.-M., RIXHON G. \& MÉRENNE-SCHOUMAKER B. (2009), Enquête socio-économique 2001, Monographie n¹0, Le mouvement pendulaire en Belgique, Les déplacements domicile-lieu de travail, Les déplacements domicileécole, Bruxelles, SPF Economie, P.M.E., Classes moyennes et Energie Direction générale Statistique et Information économique.

VERHETSEL A. et al. (2010), « Commuting in Belgian metropolitan areas. The power of the AlonsoMuth model », Journal of Transport and Land Use, 2, 3-4pp. 109-131.

VERHETSEL A. et al. (2014), Het woon-werk verkeer in Vlaanderen, Universiteit Antwerpen.

WAYENS B., JANSSENS R. \& VAESEN J. (2013), « Note de synthèse BSI. L'enseignement à Bruxelles : une gestion de crise complexe », Brussels Studies [En ligne], Notes de synthèse, $n^{\circ} 70$, mis en ligne le 29 août 2013, consulté le 02 novembre 2017, http://brussels.revues.org/1181.

ZAHAVI Y. (1974), Travel time budgets and mobility in urban areas, Washington DC, United States department of Transportation.

\section{RÉSUMÉS}

Cet article se focalise sur la mobilité quotidienne des personnes en lien avec Bruxelles au travers de deux motifs spécifiques de déplacement : les déplacements domicile-travail (DDT) et domicileécole (DDE). L'analyse de ces déplacements, très contraints d'un point de vue spatial et temporel, relève d'un double enjeu : à un niveau macro, celui de la rencontre plus ou moins heureuse entre l'offre et la demande de transport et, au niveau des ménages et des individus, celui de la capacité de produire des routines d'activités quotidiennes soutenables, dont les DDT et DDE forment bien souvent l'épine dorsale.

Au-delà de l'objet d'étude proprement dit, cet article soulève également un enjeu méthodologique important, à savoir la rupture dans la continuité des données sur les DDT/DDE suite à la disparition, en Belgique, des recensements classiques (par enquête à visée exhaustive) de la population et du logement. Avec le passage au Census, recensement basé sur des données administratives, l'information sur la mobilité des travailleurs et des écoliers s'est sensiblement appauvrie. Nous avons de ce fait eu recours à un patchwork de sources de données (enquête sur les forces de travail et Census 2011 pour les DDT ; données scolaires des Communautés françaises et flamandes, données des plans de déplacements scolaires et des prédiagnostics scolaires de la Région de Bruxelles-Capitale pour les DDE) pour reconstruire une image aussi riche que possible. 
Le constat global de l'analyse est celui d'une augmentation des distances qui traduit l'influence de divers facteurs parmi lesquels : flexibilisation du marché du travail et des carrières, situation de chômage structurel, spécialisation des activités professionnelles en ce qui concerne les DDT ; segmentation socio-spatiale de l'offre scolaire pour les DDE. La croissance de la population bruxelloise avive complémentairement l'écart entre la demande et l'offre pertinente disponible à proximité et contribue par ce biais à l'élévation des distances.

Parallèlement, le recours à l'automobile recule, dans un contexte où les freins à l'usage de la voiture se multiplient (baisse du niveau socio-économique, élévation de la congestion automobile, réduction des facilités de stationnement, réduction de l'espace public dévolu à la voiture, changement d'attitude envers la possession automobile, etc.) alors que les heures de départ s'étalent pour faire face aux congestions.

L'article conclut par l'évocation critique de diverses pistes d'actions qui soulignent l'importance de ne pas cantonner la problématique des mobilités quotidiennes à la seule question de la résolution des flux et de considérer aussi bien l'aménagement du territoire des fonctions concernées (fonctions résidentielles, scolaires et de travail) que les structures et logiques propres à chaque activité.

This paper focuses on the question of home-to-work and home-to-school commuting in Brussels. Since the latter is highly affected in terms of time and place, such an analysis is confronted with a double challenge: at a macroeconomic level the matching of supply and demand, and, for households and individuals, the capacity to put in place sustainable daily routines.

The paper also raises the fundamental question of the break in the continuity of data provision due to the suppression of the former censuses on population and housing. Nowadays those are exclusively based on administrative data, leading to a lack of data on the workers' and pupils' mobility.

This is the reason why we have chosen to use a patchwork of sources (survey on the workforce and the 2011 Census for commuting to work; data from the French and Flemish communities as well as data and school pre-diagnostics from the Brussels-Capital Region for commuting to school) so as to provide the richest possible picture.

Our analysis reveals an increase in distances due to a range of factors such as the flexibilisation of the labour and career markets, structural unemployment, specialisation of activities, etc. and the socio-spatial segmentation of school supply. The growth in population in Brussels increases the gap between supply and demand and consequently the rise in distances.

Simultaneously car transport is declining as a consequence of more and more restraints (drop in the socioeconomic level, increase in traffic congestion, changing attitudes to private car possession, etc.), while departure hours are spreading to avoid congestions.

In conclusion we critically suggest different potential actions, stressing the importance of not limiting daily commuting to the sole question of the resolution of flows and of taking into account the territorial planning of the functions in question (residence, school and work) as much as the structures and logics specific to each of those activities.

\section{INDEX}

Mots-clés : mobilité quotidienne, déplacements pendulaires, Région de Bruxelles-Capitale

Keywords : commuting to work and to school, pendular mobility, Brussels-Capital Region 


\section{AUTEURS}

\section{THOMAS ERMANS}

Centre d'Etudes Sociologiques, Université Saint-Louis - Bruxelles, thomas.ermans@usaintlouis.be

\section{CÉLINE BRANDELEER}

Centre d'Etudes Sociologiques, Université Saint-Louis - Bruxelles, celine.brandeleer@usaintlouis.be

\section{CAROLINE D'ANDRIMONT}

Institut de Gestion de l'Environnement et d'Aménagement du Territoire, Université Libre de Bruxelles, cdandrim@ulb.ac.be

\section{MICHEL HUBERT}

Centre d'Etudes Sociologiques, Université Saint-Louis - Bruxelles, michel.hubert@usaintlouis.be

\section{PIERRE MARISSAL}

Institut de Gestion de l'Environnement et d'Aménagement du Territoire, Université Libre de Bruxelles, Pierre.Marissal@ulb.ac.be 